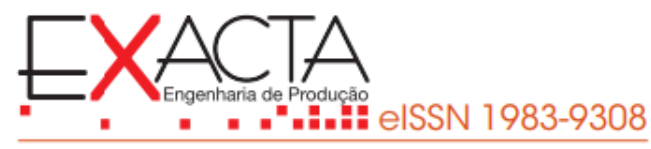

\title{
MAPEAMENTO DA PERCEPÇÃO DOS CLIENTES INTERNOS SOBRE A QUALIDADE DOS PROCESSOS: ESTUDO DE CASO NA INDÚSTRIA AUTOMOTIVA
}

\section{MAPPING THE PERCEPTION OF INTERNAL CUSTOMERS ON THE QUALITY OF PROCESSES: CASE STUDY IN THE AUTOMOTIVE IND USTRY}

Recebido em: 01 abr. 2019

Aprovado em: 13 fev. 2020

Versão do autor aceita publicada online: $13 \mathrm{fev} .2020$

Publicado online: 12 maio 2021

Como citar esse artigo - American Psychological Association (APA):

Logiúdice, R., Pacchini, A. P. T., \& Lucato, W. C. (2021, jul./set.). Mapeamento da percepção dos clientes internos sobre a qualidade dos processos: estudo de caso na indústria automotiva. Exacta, 19(3), 550-563. https://doi.org/10.5585/exactaep.2021.13378.

Submeta seu artigo para este periódico $\beta$

Dados Crossmark 


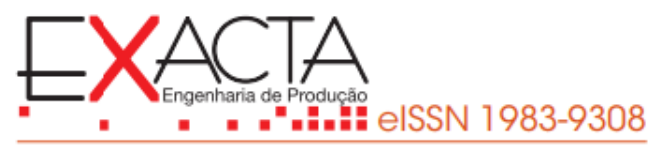

\title{
MAPEAMENTO DA PERCEPÇÃO DOS CLIENTES INTERNOS SOBRE A QUALIDADE DOS PROCESSOS: ESTUDO DE CASO NA INDÚSTRIA AUTOMOTIVA
}

\author{
MAPPING THE PERCEPTION OF INTERNAL CUSTOMERS ON THE QUALITY OF PROCESSES: CASE \\ STUDY IN THE AUTOMOTIVE INDUSTRY
}

\section{(iD) Renato Logiudice ${ }^{1}$ \\ iD Athos Paulo Tadeu Pacchini² \\ Dagner Cezar Lucato ${ }^{3}$}

\footnotetext{
${ }^{1}$ Universidade Nove de Julho - UNINOVE. São Paulo, SP.

Doutor em Engenharia de Produção renato.logiudice@uni9.pro.br

${ }^{2}$ Universidade Nove de Julho - UNINOVE Doutor em Engenharia de Produção athos@uni9.pro.br

${ }^{3}$ Universidade Nove de Julho - UNINOVE. São Paulo, SP.

Doutorado em Engenharia de Produção (Conceito CAPES 4).

Universidade Metodista de Piracicaba, UNIMEP, Brasil.

wlucato@uni9.pro.br
}

Recebido em: 01 abr. 2019

Aprovado em: 13 fev. 2020
Resumo: Este trabalho tem como objetivo avaliar a percepção dos clientes em relação aos processos internos de uma indústria metalúrgica de grande porte dedicada à manufatura de autopeças. A avaliação foi feita considerando os seguintes aspectos: conformidade do produto, prazos e atendimento. Diversos estudos têm analisado a cadeia de valor, mas poucos tratam sobre a identificação dos pontos a serem melhorados, de forma colaborativa, dentro da empresa. Neste trabalho, foi desenvolvido um estudo de caso em uma indústria pertencente à cadeia logística automotiva. Por meio dele, pontos críticos foram encontrados nos elos cliente-provedor interno da cadeia de valor. Nessa avaliação, setores considerados como relevantes nesse ramo de atividade, como ferramentaria e compras, apresentaram os piores resultados no que se refere aos prazos. Já a ferramentaria se mostrou- deficiente no quesito atendimento na prestação dos serviços.

Palavras-chave: Processos internos. Cadeia de valor. Clientes internos.

Abstract: This work aims to evaluate the customers perception of the internal processes of a large metallurgical industry dedicated to the manufacture of auto parts. The evaluation was made considering the following aspects: product compliance, deadlines and attendance. Several studies have analyzed the value chain, but few have proposed the identification of the points to be improved collaboratively within the company. In this work, a case study was carried out and critical points were found in the internal clientprovider links of the value chain. In this evaluation, sectors considered as relevant in this field of activity, such as tooling and purchasing, presented the worst results in performance factors of deadlines. Tooling was shown to be deficient in the service provision.

Keywords: Internal processes. Value chain. Internal clients. 


\section{Introdução}

A vantagem competitiva tem nascentes nas muitas atividades distintas que a empresa desenvolve e que dá apoio à cadeia de suprimentos na geração do produto ou serviço. Cada uma dessas atividades contribui para o resultado e cria uma base para a diferenciação (Vargas, Montilla \& Jabour, 2018).

Pode-se considerar que a linha de produção, de uma empresa, é composta por um ou mais processos concatenados. Cada um desses processos deverá entregar para o estágio seguinte componentes ou serviços com os requisitos de qualidade estabelecidos de modo satisfatório. Para avaliar a necessidade de melhorar o desempenho dos processos, convém examinar os resultados dos produtos ou serviços de cada um dos processos. A qualidade dos produtos e serviços oferecidos pelos provedores internos pode ser monitorada pelos seguintes indicadores de desempenho: atendimento aos prazos de entregas acordados e entrega dos pedidos de acordo com as especificações e o atendimento colaborativo (Aharonovitz, Vieira \& Suyama, 2018).

Com a utilização de uma escala Likert pode-se quantificar uma variável intangível, como nos casos do atendimento colaborativo, assim, a avaliação do desempenho dos provedores internos na empresa passa a ser suscetível de análise. Diversos estudos têm analisado as cadeias de valores, mas poucos sugerem a aplicação de métodos para operacionalizar o monitoramento da qualidade no atendimento e prazos nos elos clientes-provedores, no âmbito interno da organização, (ArlbjØrn \& Freytag, 2013; Mayer, Borchardt \& Pereira, 2016) A escassez de estudos práticos com relação a avaliação dos clientes internos, estimulou o presente trabalho no sentido de constatar como isso é operacionalizado num caso real em uma indústria do setor automotivo. Já na área de serviços, diversos estudos são relatados e com resultados válidos da sua aplicabilidade, porém, essa possibilidade nem sempre se confirma, quando a estrutura do sistema de gestão não dispõe de meios para a realização do monitoramento dos pontos críticos dos processos envolvidos, (Piercy \& Rich, 2009). Ainda assim, uma relação de colaboração entre os donos dos processos internos envolvidos permite melhorar o desempenho como um todo. Conforme Xu, Goedezebuure e Heijden (2006), na percepção do cliente, benefícios sociais, relativos à qualidade no atendimento proporcionado pelo fornecedor, têm um impacto positivo na satisfação, pois maiores níveis de confiança nesse relacionamento resultarão em menor ansiedade durante às interações

Assim, o objetivo do presente estudo foi avaliar os aspectos da qualidade do atendimento, dos prazos de entrega e da conformidade das características do item produzido, isso sob a percepção dos clientes internos dos processos operacionais. Com o mapeamento dos resultados, procurou-se identificar quais são os processos que ocasionam mais problemas no âmbito interno da empresa. Para 
suportar essa análise, recorreu-se a um estudo de caso para realizar o mapeamento dos processos internos (produtivos e de apoio) da empresa em questão, bem como a identificação dos seus clientes e provedores. Para isso, aplicou-se um questionário aos responsáveis pelos processos, no qual notas deveriam ser atribuídas aos seus provedores com relação aos indicadores de prazo e à conformidade com as especificações do item fabricado e qualidade no atendimento.

\section{Revisão da literatura}

Para se compreender o elo existente entre a vantagem competitiva e as várias atividades executadas na cadeia de valor de uma empresa, bem como o modo como elas interagem, tem-se os estudos de Michael Porter (1985). Para o autor, a cadeia de valor deu uma base para cada atividade dentro da organização, para possibilitar adicionar valor ao cliente. Nesse caso, a logística tem um papel central na criação de valor para o cliente, dentro e fora dos limites das empresas.

Conforme Bowersox, Closs e Cooper (2006), a satisfação é alcançada quando ocorre a criação de valor ao cliente, de modo que suas expectativas em relação aos requisitos qualidade e disponibilidade são atendidas. Entretanto, Khan e Mentzer (1996) e Christopher (2016) observaram que os profissionais de gestão da logística focam na integração de seus parceiros externos apenas, não dando a mesma atenção, sob o ponto de vista interdepartamental, ao contexto clientes e fornecedores internos.

A integração da cadeia de valor da empresa, notadamente no controle dos lead times, propicia melhorias no desempenho logístico (Hilsdorf, Rotondaro \& Pires, 2009; Christopher, 2016). Uma das razões da complexidade do gerenciamento e da avaliação da cadeia de suprimentos é a dificuldade em se definir as fronteiras do sistema a ser mensurado. Outro problema que merece atenção é a falta de ligação entre estratégia e indicadores de desempenho (Carter, Rogers \& Choi, 2015).

De acordo com Grahan (2018), a elaboração de um produto ou serviço para um cliente é realizada pela sequência concatenada de atividades em um ou mais processos interligados. Existe toda uma interação entre clientes e provedores internos, mas o objetivo principal é a produção de um bem ou serviço para o cliente final.

Nesse sentido, o objetivo principal da gestão é manter e melhorar os padrões de trabalho para a contínua satisfação dos clientes, internos e externos. Apesar de direcionar para as atividades do dia a dia da organização, não é bom perder a perspectiva das necessidades e expectativas do cliente externo. Medidas e métricas são frequentemente propostas, independentemente do produto, da indústria, ou do tipo de cliente, para atingir o nível de serviço exigido (Grahan, 2018).

A qualidade dos produtos e serviços prestados pelos provedores pode ser avaliada por indicadores de desempenho, tais como a quantidade ou percentagem de entregas realizadas dentro do 
prazo, o recebimento do pedido de acordo com as especificações ou a percentagem de entregas devolvidas parcial ou integralmente. Com a mudança de cenário, onde a disputa não acontece entre uma empresa e sua concorrente, mas entre as suas cadeias de suprimentos, a avaliação do desempenho logístico assume papel relevante na melhoria da cadeia como um todo (Aharonovitz, Vieira \& Suyama, 2018). Desperdícios nos processos internos comprometem a produtividade da empresa. Como desperdícios, pode-se citar filas e excesso de material, produção além do programado, tempo de espera, movimentação de material, preparações de máquina, processos errados e produção de peças defeituosas (Rossetti, Barros, Tódero, Denicol, \& Camargo, 2008).

Atualmente, a análise da interação dos processos é mais complexa quando se refere à integração, ainda mais com o aumento da terceirização de serviços, embora esta tenha a vantagem da redução dos custos. Os requisitos na interação clientes-provedores continuam sendo praticamente os mesmos, ou seja: qualidade no atendimento, características do item, prazo e preço (Vieira \& Rodrigues, 2011).

Em relação ao desenvolvimento teórico exposto, a integração logística ainda apresenta alguns desafios. Zhang, Guo e Liu (2018), por exemplo, apontam que a conexão entre manufatura e vendas ainda é mais forte do que entre manufatura e compras. Isso indica que, já internamente, a integração está sendo parcial, dificultando a visão da cadeia como um todo. Isso pode ser, segundo o autor, uma consequência das políticas orientadas ao consumidor, que acabam dando maior ênfase a jusante.

De acordo com Daugherty, Ellinger e Gustin, (1997), várias empresas constataram que, se as atividades logísticas estiverem coordenadas com propósitos de melhorias, os custos totais podem ser reduzidos, o serviço ao cliente pode ser melhorado, e os conflitos interdepartamentais podem ser substancialmente diminuídos.

Em se tratando de melhorias, os sistemas de informação são utilizados com o objetivo de otimizar o desempenho de uma organização, por meio de hardwares e softwares para o gerenciamento das operações, seja em uma só empresa ou em toda uma cadeia de suprimentos. Para melhorar o fluxo de informação dentro da cadeia, os parceiros devem ter um conjunto de medidas em comum e uma confiança mútua que seja suficiente para compartilhar essa informação (Petersen, Ragatz, \& Monczka, 2005; Handfield \& Bechtel, 2002). Por outro lado, as práticas colaborativas podem ser impulsionadas por modelos de cadeia de suprimentos, como a Efficient Consumer Response, ou Resposta Eficiente do Consumidor, para melhorar o desempenho por meio do compartilhamento de informações (Bailey \& Francis, 2008).

Segundo Reagan (2011) e Antonioli e Calarge (2013), o Lean Service, ou Serviço Enxuto, é frequentemente usado em departamentos operacionais para gerar benefícios. No entanto, deveria ser aplicado também nos departamentos de suporte, em áreas que possuem características de serviços, uma vez que tem impacto significativo sobre a qualidade. Ainda segundo os autores, todas as etapas 
interligadas ao processo interno da empresa devem ser continuamente melhoradas para oferecer e sustentar o serviço superior por meio de atividades interconectadas que geram benefícios nas atividades subsequentes.

Uma constatação empírica conduzida por Parasuraman, Zeithaml e Berry (1985) revela que, independentemente do serviço, os clientes usam os mesmos critérios gerais para chegar a uma avaliação sobre a qualidade do serviço recebido. Correspondentemente, o estudo empírico de Van Montfort et al. (2000) revelou que a satisfação pode ser prevista pelo desempenho de serviço percebido pelos clientes.

As pesquisas de Gremler e Gwinner (2000) indicam que a afinidade e a harmonia do clientefornecedor estão positivamente relacionadas à satisfação com o prestador de serviços. Analogamente, Hennig-Thurau, Guinner e Gremler, (2002), assumem que existe uma relação positiva entre o benefício social e a satisfação do cliente.

Diante dessas constatações, a proposição para este estudo se apresenta como: O mapeamento da satisfação dos clientes internos pode revelar pontos críticos para ações corretivas que visam a melhoria da qualidade nas interfases clientes fornecedores internos.

\section{Procedimentos metodológicos}

No presente trabalho, utilizou-se o método hipotético-dedutivo proposto por Lakatos e Marconi (2017), uma vez que o grande estímulo, para essa pesquisa, foi avaliar a percepção dos clientes internos da cadeia logística quanto ao desempenho dos provedores da empresa pesquisada. Isso resultou em um estudo de caso com observação direta extensiva por meio da realização de entrevista semiestruturada e da aplicação de formulário. De acordo com Yin (2010), o estudo de caso "é uma investigação empírica que analisa um fenômeno contemporâneo dentro de um contexto da vida real", no qual múltiplas fontes e evidências são usadas. Esse tipo de procedimento envolve o estudo profundo e exaustivo de um ou poucos objetos, permitindo o seu amplo e detalhado conhecimento.

Segundo Ellram (2006), o método do estudo de caso vem se tornando bem aceito, principalmente em pesquisas relacionadas à gestão de operações e à cadeia de suprimentos, por se tratar de uma forma legítima e válida de adicionar subsídios a esse campo de conhecimento, já que outros tipos de abordagem não conseguem suprir essa necessidade.

Para realizar o estudo de caso, que suportou o desenvolvimento aqui proposto, estabeleceramse os seguintes critérios para seleção da empresa: a) deveria ser uma empresa de médio ou grande porte para garantir um nível de processos internos devidamente segmentados, o que é fundamental para a realização da presente avaliação; b) ser uma empresa com processos internos estruturados de maneira a possibilitar a sua completa compreensão e identificação das fronteiras entre os diversos 
agentes que atuam nos referidos processos; e c) a empresa deveria garantir aos pesquisadores amplo acesso aos diferentes clientes internos, para que as informações necessárias pudessem ser adequadamente colhidas.

Como decorrência desses critérios, a empresa selecionada como objeto deste estudo pertence ao segmento metalúrgico, e é especializada na fabricação de peças forjadas e usinadas de alta precisão, em ligas de metais não-ferrosos, direcionadas ao segmento automotivo. Além disso, a empresa possui em torno de 600 funcionários e um parque industrial de $14.000 \mathrm{~m}^{2}$, localizado na cidade de São Paulo. Os principais macroprocessos de fabricação mapeados na empresa estudada (o que impacta na cadeia de valor) são mostrados na Figura 1, conforme a sua sequência.

O método utilizado para a coleta dos dados foi o depoimento dos responsáveis pelos processos produtivos da empresa, obtido por meio de uma entrevista semiestruturada e do preenchimento de um formulário, de modo a identificar a estrutura interna da empresa, ou seja, sua cadeia de suprimentos, seus membros-chave, seus principais processos de negócio, seu nível de integração dos processos, as práticas utilizadas, seus fluxos de informação e materiais, além de outros dados que pudessem ser relevantes para o objetivo da pesquisa.

Figura 1

Mapeamento da Estrutura dos Macroprocessos

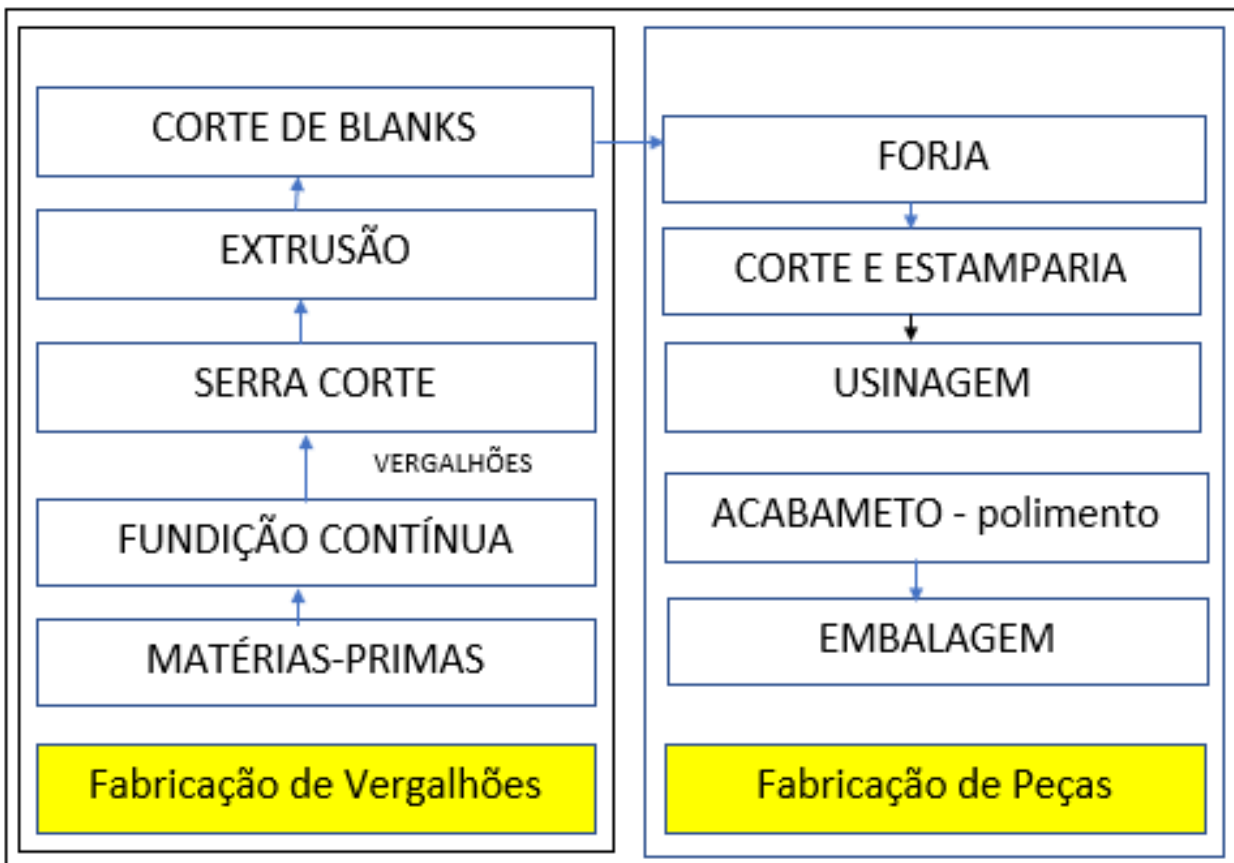

Fonte: Os autores (2019)

O objetivo desse procedimento foi mapear e medir o grau de integração dos processos logísticos internos com seus usuários. 
As entrevistas realizadas foram conduzidas com o auxílio de um questionário, em que se utilizou a escala Likert, variando de 1 a 5 pontos, com a seguinte graduação de medição para cada uma das afirmativas propostas acerca da efetiva prática dos fatores de integração considerados: (1) péssimo, (2) ruim, (3) regular, (4) bom e (5) ótimo. A utilização da escala de Likert teve como objetivo facilitar a obtenção das respostas e a interpretação das informações obtidas.

A qualidade em serviços é a percepção entre os requisitos esperados, ou que foram acordados, e os resultados recebidos (Gronroos, 1984; Kotler, 2000; Lehtinen \& Lehtner, 1982; Lewis \& Booms, 1983; Parasuraman et al., 1988; Zeithaml,1987). Os indicadores utilizados na pesquisa tiveram como base o método SERVQUAL, que representa um dos mais amplamente utilizados instrumentos de medição da qualidade do serviço. Ele forneceu aos pesquisadores um meio de fazer essa medição com base em cinco dimensões: aspectos tangíveis, confiabilidade, responsividade, garantia e empatia (Parasuraman et al. 1988).

Considerando que o conceito de serviço ao cliente interno da empresa está relacionado diretamente às atividades do sistema logístico na cadeia de suprimentos, foram utilizados, como arcabouço teórico para delimitação das variáveis a serem utilizadas na pesquisa, os processos-chave denominados "gestão da demanda" e "atendimento de pedidos" (Christopher, 2016), os quais envolvem basicamente subprocessos de natureza logística, tais como: processamento de pedidos, gestão de estoques, embalagem e manuseio de produtos, transporte e entrega, e gestão da informação sobre o pedido.

A partir dessas atividades, foram definidos os três fatores de integração a serem utilizados como referência para a construção do instrumento de pesquisa. São eles: (1) características de desempenho do prazo de entrega dos produtos e serviços, conforme programação da produção estabelecida; (2) conformidade dos produtos de acordo com as especificações requeridas; e (3) qualidade do atendimento na prestação dos serviços. Para as três características, foram calculados seus desviospadrão e as médias para embasar as conclusões desse trabalho.

Para o mapeamento da percepção dos clientes internos sobre a qualidade dos processos, foram elaboradas algumas tabelas para cada um dos três fatores de integração. Nelas, foram elencados os setores produtivos, bem como a avaliação com os pontos obtidos dos seus clientes internos, as médias e os desvios-padrão. Os resultados das médias e dos desvios foram sinalizados com cores que correspondem as pontuações estabelecidas na escala Likert, para facilitar a gestão à vista. Tais tabelas são demonstradas no próximo tópico.

\section{Resultados e discussões}


Cada cliente interno, responsável pelo processo analisado da empresa, respondeu às questões da pesquisa sobre a qualidade no prazo de entrega dos produtos e serviços, a conformidade dos produtos e serviços recebidos e a qualidade no atendimento. As pontuações obtidas de cada um dos provedores internos da empresa foram tabuladas e estão representadas nos Quadros 1, 2 e 3.

Para as pontuações de cada provedor, foram calculados as médias e os desvios-padrão, para constatar se a média representa uma unanimidade na avaliação dos clientes e se existe uma variação significativa.

Da análise dos resultados apresentados no Quadro 1, que se trata do prazo de entrega dos produtos e serviços,, constatou-se que o setor de compras apresenta uma pontuação média com conceito ruim, e um desvio padrão que mostra pouca dispersão desse conceito, o que está de acordo com as afirmações de Zhang, Guo e Liu (2018). Isso demonstra a necessidade de se implementar uma ação de melhoria para a diminuição desses possíveis gargalos no fluxo produtivo, conforme enfatizado por Rossetti et al. (2008). Constatou-se ainda que vários setores possuem uma dispersão das pontuações elevada, principalmente devido à péssima avaliação de um dos clientes interno, fato este que merece atenção especial, como confirmado no estudo de Piercy e Rich (2009).

\section{Quadro 1}

Característica da Qualidade: Prazo de Entrega dos Produtos ou Serviços

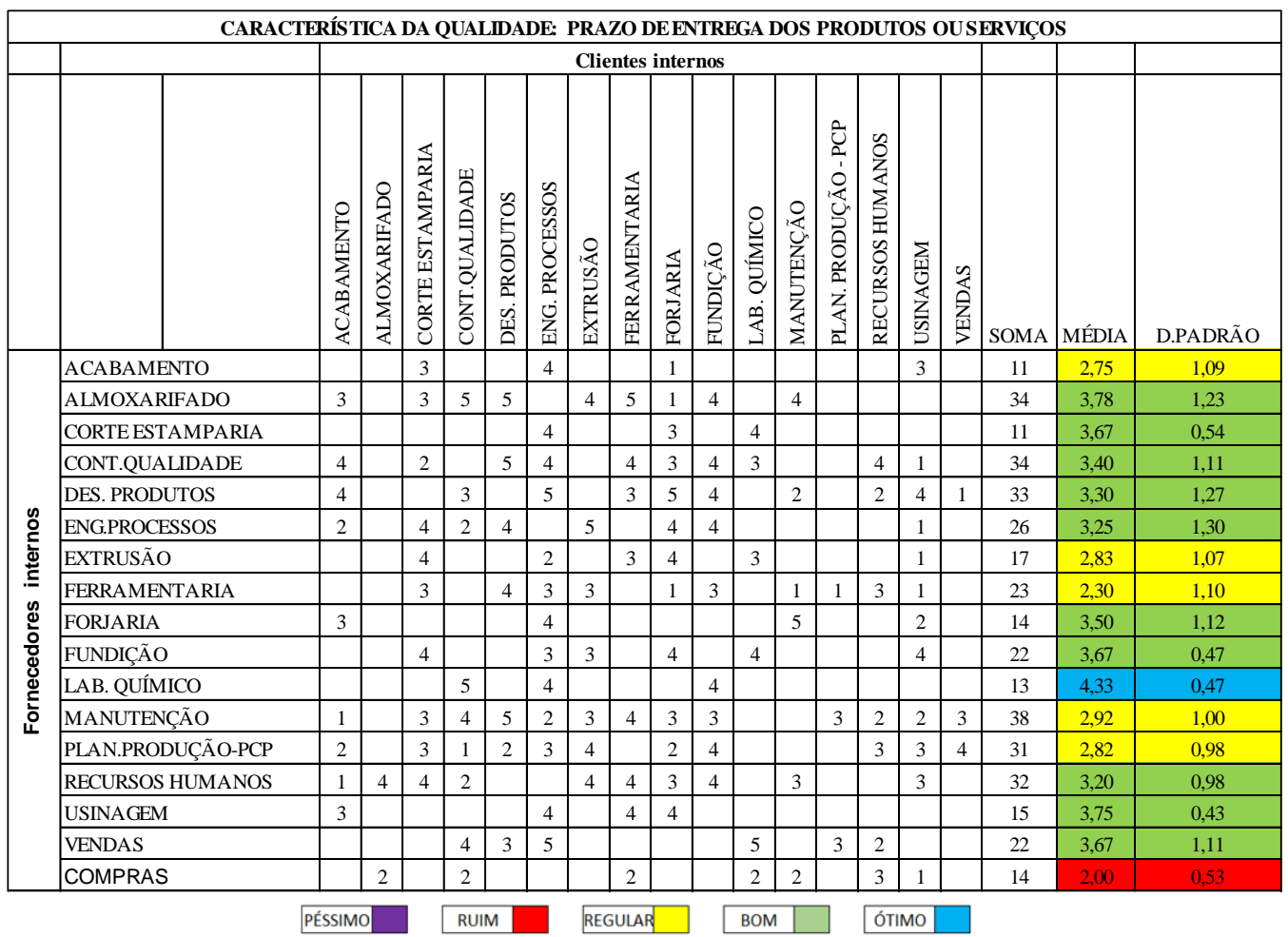

Fonte: Os autores (2017). 
Já com base na análise do Quadro 2, que se refere à conformidade dos produtos e serviços, contatou-se que existem setores, como o Laboratório Químico e a Engenharia de Processos, que apresentam uma excelente pontuação, o que denota que a empresa possui um corpo técnico competente em suas atribuições e também uma estrutura laboratorial compatível com as exigências dos processos envolvidos. Os setores de PCP (Planejamento e Controle da Produção) e extrusão apresentaram uma pontuação baixa, tanto no requisito prazo de entrega como no atendimento dos requisitos de qualidade estabelecidos para os produtos e serviços esperados pelos seus clientes internos. Isso denota falhas constantes na programação e na qualidade dos produtos. Observou-se que o setor de corte fez uma boa avaliação dos prazos de entrega do setor de extrusão, porém, os aspectos do atendimento das características do produto receberam uma pontuação baixa. Os setores de Laboratório Químico e Engenharia de Processos obtiveram uma excelente pontuação, justificando os investimentos que a empresa fez nos recursos humanos envolvidos e também nas instalações e na tecnologia da informação.

\section{Quadro 2}

Característica da Qualidade e Conformidade dos Produtos ou Serviços

\begin{tabular}{|c|c|c|c|c|c|c|c|c|c|c|c|c|c|c|c|c|c|c|c|c|}
\hline \multicolumn{21}{|c|}{ CARACTERÍSTICA DA QUALIDADE: CONFORMIDADE DOS PRODUTOS OU SERVIÇOS } \\
\hline & & & & & & & & Clie & ntes & inter & nos & & & & & & & & & \\
\hline & & 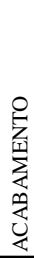 & 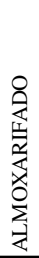 & 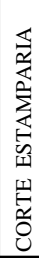 & 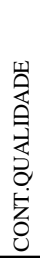 & 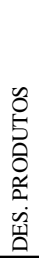 & 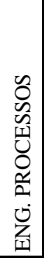 & 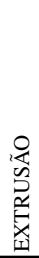 & 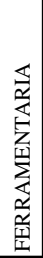 & 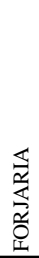 & 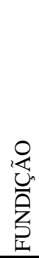 & 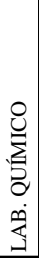 & 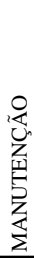 & 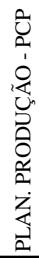 & 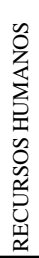 & 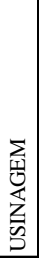 & $\sum_{\substack{1\\
}}^{\sum_{1}}$ & SOMA & MÉDIA & D.PADRÃO \\
\hline \multirow{17}{*}{ 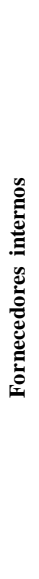 } & ACABAMENTO & & & 5 & & 5 & 4 & & 5 & 2 & & 4 & & & & 2 & & 27 & 3,86 & 1,25 \\
\hline & ALMOXARIFADO & 4 & & 4 & 5 & & & 4 & & 3 & 2 & & 4 & & & 3 & & 29 & 3,63 & 0,86 \\
\hline & CORTE ESTAMPARIA & & & & & & 3 & & & 1 & & 4 & & & & 3 & & 11 & 2,75 & 1,09 \\
\hline & CONT.QUALIDADE & 4 & 5 & 4 & & 4 & 4 & & 4 & 3 & 4 & 3 & & & 4 & 1 & & 40 & 3,64 & 0,98 \\
\hline & DES. PRODUTOS & 4 & & & 4 & & 5 & & 4 & 4 & 4 & & 3 & & 3 & 2 & 4 & 37 & 3,70 & 0,78 \\
\hline & ENG.PROCESSOS & 4 & & 4 & 4 & 5 & & 5 & & 4 & 4 & & & & & 2 & & 32 & 4,00 & 0,87 \\
\hline & EXTRUSÃO & & & 3 & & & 3 & & & & & 3 & & & & 1 & & 10 & 2,50 & 0,87 \\
\hline & FERRAMENTARIA & & & 4 & & 4 & 5 & 3 & & 4 & 4 & & 2 & 2 & 4 & 3 & & 35 & 3,50 & 0,92 \\
\hline & FORJARIA & 2 & & & & & 4 & & & & & 5 & & & & 3 & & 14 & 3,50 & 1,12 \\
\hline & FUNDIÇÃO & & & 4 & & 5 & 4 & & & & & 4 & & & & 1 & & 18 & 3,60 & 1,36 \\
\hline & LAB. QUÍMICO & & & & 5 & & 5 & & & & 5 & & & & & & & 15 & 5,00 & 0,00 \\
\hline & MANUTENÇÃO & 4 & & 3 & 4 & 4 & 3 & 3 & 3 & 3 & 4 & & & 3 & 4 & 3 & 5 & 46 & 3,54 & 0,63 \\
\hline & PLAN.PRODUÇÃO-PCP & 4 & & 4 & 2 & 3 & 2 & 3 & & 2 & 3 & & & & 3 & 1 & 5 & 32 & 2,91 & 1,08 \\
\hline & RECURSOS HUMANOS & 3 & 5 & 4 & 3 & & & 4 & 4 & 4 & 5 & 4 & 4 & & & 1 & & 41 & 3,73 & 1,05 \\
\hline & USINAGEM & 3 & & & & & 4 & & 4 & 5 & & & & & & & & 16 & 4,00 & 0,71 \\
\hline & VENDAS & & & & 4 & & 2 & & & & & 5 & & 2 & 2 & & & 15 & 3,00 & 1,26 \\
\hline & COMPRAS & 2 & 3 & & 3 & & & & 4 & & & 4 & 4 & & 4 & 3 & & 27 & 3,38 & 0,70 \\
\hline & & & & & & JIM & & & REG & JLAR & & & BO & & & & & & & \\
\hline
\end{tabular}

Fonte: Os autores (2017).

A pontuação quanto à qualidade no atendimento sinalizou um estágio de amadurecimento dos relacionamentos pessoais entre os setores da empresa. Na administração participativa, a harmonização dos relacionamentos leva a construir a cultura organizacional voltada à colaboração, o que está de 
acordo com Xu, Goedezebuure e Heijden (2006). Da análise dos resultados contidos no Quadro 3, que se refere à qualidade do atendimento, o setor de ferramentaria também obteve uma pontuação baixa, porém, devido às pontuações dos setores de apoio à produção, como o PCP e a manutenção, constituindo um problema de estabelecimento de prioridades e deve-se deixar isso bem claro para o elo cliente-provedor e interno como característica nas negociações internas de requisições de serviços e apoio. Conforme Porter (1985), as organizações devem olhar para cada atividade em sua cadeia de valor e avaliar se elas têm uma vantagem competitiva real. Se não tiverem, então talvez devam considerar a terceirização dessa atividade.

\section{Quadro 3}

Característica da Qualidade: Atendimento

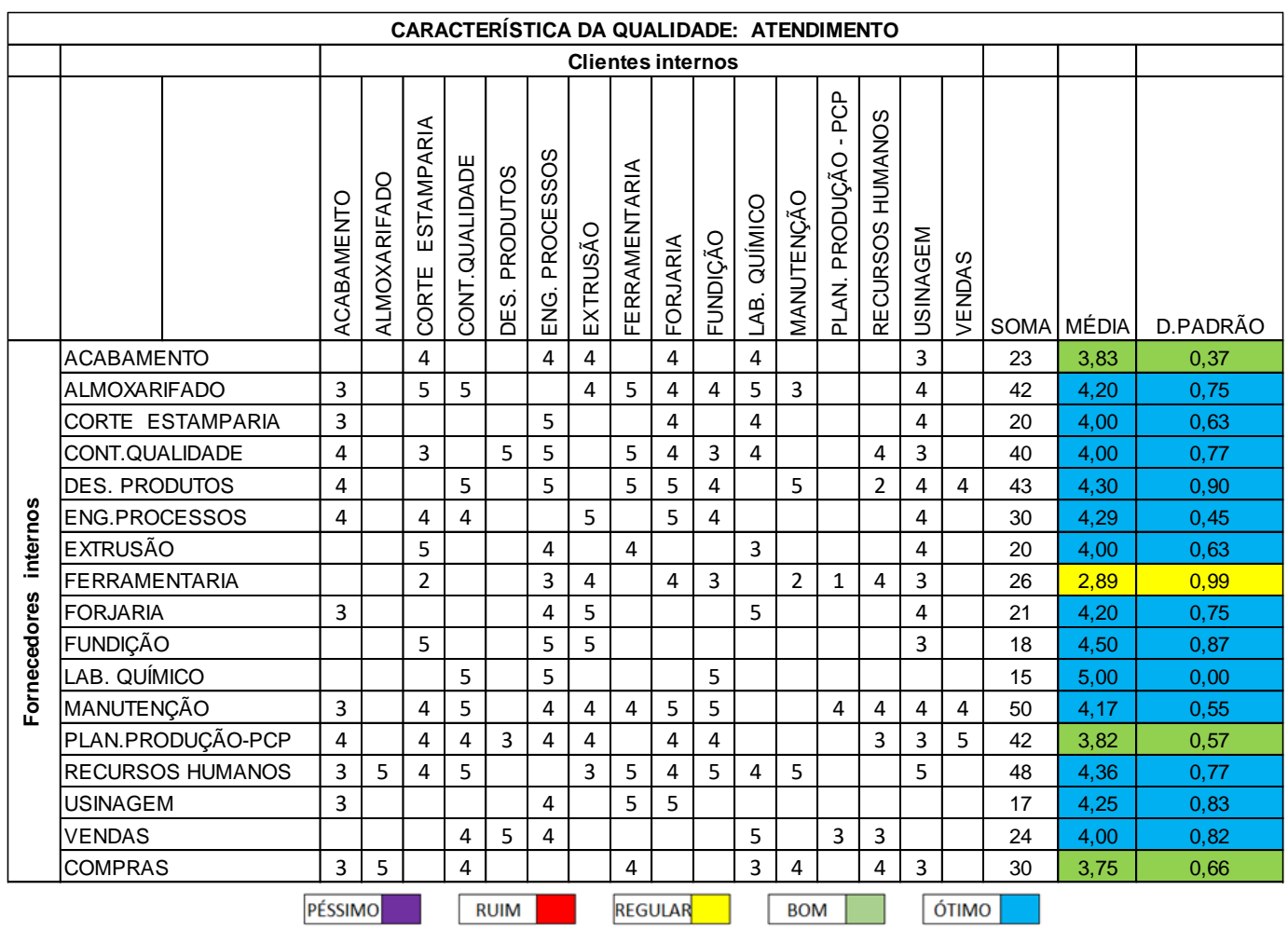

Fonte: Os autores (2017).

\section{Considerações finais}

Com este estudo, constatou-se que há diversos estudos na literatura sobre melhorias na cadeia de suprimentos e sua integração. Entretanto, poucos abordam os métodos para identificação de problemas. No nível das operações internas à empresa, os aspectos tangíveis são facilmente identificados, e as ações corretivas, implementadas. Porém, as características intangíveis da qualidade na prestação de serviços de apoio, e mesmo nos elos produtivos da cadeia de valor, acabam passando 
despercebidas. Por essa razão, os indicadores que possibilitam sinalizar essas não-conformidades são importantes para auxiliar os gestores nas tomadas de decisões.

O estudo de caso aqui desenvolvido, embora não possa ser generalizado, é adequado para a aplicação em diversas empresas, pela sua simplicidade e pelo seu baixo custo. Além disso, com a ajuda das novas tecnologias da informação, favorecerá a reorganização e a visão detalhada dos processos.

Considerando as percepções dos clientes internos avaliados, constatou-se que os setores de ferramentaria e compras apresentam as piores notas em relação ao prazo de entrega e à conformidade dos produtos ou serviços. O mesmo vale para os setores de PCP (Planejamento e Controle da Produção), corte e estamparia e extrusão. No que se refere ao atendimento, a ferramentaria foi a que obteve pior avaliação nos processos internos.

Com base na análise realizada, pode-se assegurar que este trabalho traz contribuições tanto teóricas como práticas para a Gestão de Operações. Para o campo de conhecimento, os resultados aqui encontrados contribuem com a literatura na medida em que agregam novas informações e conclusões que, até então, não haviam sido devidamente exploradas na bibliografia que trata do monitoramento de aspectos da qualidade nos elos clientes fornecedores internos. Para a prática, este trabalho apresenta, aos gestores dos processos internos, uma ferramenta simples e econômica que permite identificar pontos que precisam de atenção a fim de implantar melhorias nos processos internos da empresa.

Como toda pesquisa, está também tem limitações. Primeiramente, por se tratar de um estudo de caso único, não se pode fazer generalizações. Ademais, a aplicação foi feita em uma empresa do setor automotivo que, em geral, possui processos internos bem estruturados. Portanto, para minimizar tais entraves, recomenda-se realizar estudos futuros nos quais os conceitos aqui apresentados sejam aplicados a um número maior de empresas pertencentes a diferentes setores industriais e de prestação de serviços.

\section{Referências}

Aharonovitz, M.C.S., Vieira, J.G.V. \& Suyama, S.S. (2018). How logistics performance is affected by supply chain relationships. The International Journal of Logistics Management, Vol. 29, No. 1 , pp.284-307.

Antonioli. A., Calarge, F. A. (2013). A abordagem do lean servisse, uma revisão de literatura baseada em análise bibliométrica apresentada no XXXIII Encontro Nacional de Engenharia de Produção, A Gestão dos Processos de Produção e as Parcerias Globais para o Desenvolvimento Sustentável dos Sistemas Produtivos, Salvador, BA, Brasil, 08 a 11.

Arlb $\varnothing$ rn, J. S., Freytag, P. V. (2008). Evidence of lean: a review of international peer-reviewed journal articles. European Business Review. Vol. 25, No. 2, pp. 174-205. 
Bailey, K., Francis, M. (2008). Managing information flows for improved value chain performance. International Journal of Production Economics, Vol. 111, No. 1, pp. 2-12.

Bowersox, D. J.; Closs, D. J., Cooper, M. (2006). Gestão logística de cadeias de suprimento. Porto Alegre: Bookman.

Carter, C.R., Rogers, D.S. \& Choi, T.Y. (2015). Toward the theory of the supply chain. Journal of Supply Chain Management. Vol. 51, No. 2, pp. 89-97.

Christopher, M. (2016). Logistics and supply chain management. 5.ed. London: Financial Times Publishing.

Daugherty, P. J., Ellinger, A. E., Gustin, C. M. (1996). Integrated logistics: achieving logistics performance improvements. Supply Chain Management. Vol.1, n.3, pp. 25-33.

Ellram, L. M. (2006). The implementation of target costing in the United States: theory versus practice. The Journal of Supply Chain Management, Winter, pp. 13-25.

Grahan, S. (2018). Antecedents to environmental supply chain strategies: The role of internal integration and environmental learning. International Journal of Production Economics, Vol. 197, pp. 283-296.

Gremler, D.D., Gwinner, K.P. (2000). Customer-Employee Rapport in Service Relationships. Journal of Service Research, Vol. 3, pp. 82-104.

Gronroos, C. (1984). A Service Quality Model and Its Market Implications. European Journal of Marketing, 18 (4), 36-44.

Handfield, R. B., Bechtel, C. (2002). The role of trust and relationship structure in improving supply chain responsiveness. Industrial Marketing Management, Vol. 31, No. 4, pp. 367-382.

Hennig-Thurau, T., Guinner, K. P. ; Gremler, D.D. (2002). Understanding Relationship Marketing Outcomes An Integration of Relational Benefits and Relationship Quality. Journal of Service Research, Volume 4, No. 3, pp.230-247

Hilsdorf, W. C., Rotondaro, R. G., Pires, S. R. I. (2009). Integração de processos na cadeia de suprimentos e desempenho do serviço ao cliente: um estudo na indústria calçadista de Franca. Gestão \& Produção, v. 16, n. 2, pp. 232-244.

Kahn, K. B.; Mentzer, J. T. (1996). Logistics and interdepartmental integration, International Journal of Physical Distribution \& Logistics Management, MCB University Press, v. 26, n. 8, p. 6-14.

Kotler, P. (2000). Marketing Management. Upper Saddle River, NJ: Prentice-Hall Inc.

Lakatos, E. M., Marconi, M. (2017). Fundamentos da metodologia científica. (7ạ. Ed.) São Paulo: Atlas

Likert, R. (1932) A technique for the measurement of attitudes. Archives of Psychology. n. 140, p. 4453.

Lehtinen, U., Lehtner, J.R. (1982). Service Quality-A Study of Dimensions. Unpublished Working Paper. Helsinki: Service Management Institute, pp. 439-460. 
Lewis, R.D., Booms, B.H. (1983). The Marketing Aspects of Service Quality.

Mayer, J.A., Borchardt, M., Pereira, G.M. (2016). Methodology for the collaboration in supply chains with a focus on continuous improvement. Ingeniería e Investigaclón vol. 36 n. ², august -pp. 51-59.

Parasuraman, A., Zeithaml, V. A, \& Berry, L. L. (1988). SERVQUAL: A Multiple-Item Scale for Measuring Consumer Perceptions of Service Quality. Journal of Retailing, 64, 12-40.

Piercy, N., Rich, N. (2009). Lean transformation in the pure service environment: The case of the call service centre. International Journal of Operations \& Production Management, Vol. 29 No. 1, pp. 54-76.

Petersen, K., Ragatz, G.L., Monczka, R.M. (2005). An examination of collaborative planning effectiveness and supply chain performance. Journal of Supply Chain Management, Vol. 41, No. 2, pp. 14-25.

Porter, Michael E. (1985). Competitive advantage. New York: The Free Press.

Reagan, R. (2011). Department of Financial Service Embraces Lean. Government Finance Review.

Rossetti, E.K., Barros, M.S., Tódero, M.; Denicol, S., Camargo, M.E. (2008). Sistema Just in Time: conceitos imprescindíveis. Revista Qualit@s. Vol.7, n. 2, pp. 1-6.

Vargas, J.R.C., Montilla, C.E.M, Jabour, A.B.L.S. (2018). Enablers of sustainable supply chain management and its effect on competitive advantage in the Colombian context. Resources, Conservation and Recycling, Vol. 139, pp. 237-250.

Van Montfort, K., Masurel, E., Van, R. I. (2000). Service Satisfaction: An Empirical Analysis of Consumer Satisfaction in Financial Services. The Service Industries Journal, Vol. 20 pp., 80-94.

Vieira, C. L. S., Rodrigues, C.M.T. (2011). Uma perspectiva sobre o desenvolvimento do conceito de logística apresentado no XXXI Encontro Nacional de Engenharia de Produção Inovação Tecnológica e Propriedade Intelectual: Desafios da Engenharia de Produção na Consolidação do Brasil no Cenário Econômico Mundial, Belo Horizonte, MG, Brasil, 04 a 07.

Xu, Y., Goedezebuure, R., Heijden, B.V. (2006). Customer Perception, Customer Satisfaction, and Customer Loyalty Within Chinese Securities Business. Journal of Relationship Marketing, Vol.5. pp. 79-104.

Yin, Robert K. (2010). Estudo de caso - planejamento e métodos. (4ạEd. p.30). Porto Alegre: Bookman.

Zhang, Y., Guo, Z., Lv, J., Liu, Y. (2018). A framework for smart production-logistics systems based on CPS and industrial IOT. IEEE Transactions on Industrial Informatics, Vol. 14, No. 9, pp. 40194032. 